\title{
Estimation of height for persons in pictures
}

\author{
KINUYO KATO \\ Nagoya University, Nagoya, Japan \\ and \\ ATSUKI HIGASHIYAMA \\ Ritsumeikan University, Kyoto, Japan
}

\begin{abstract}
We report three experiments in which subjects estimated the height of persons seen in pictures. In Experiment 1, 50 realistic photographs were used as targets. The correlation between mean estimated height and actual height was .92, and target's sex and the ratios of head to trunk, which included horizontal ratio (i.e., a ratio of head width to shoulder width) and vertical ratio (i.e., a ratio of head height to stature), were found to be effective on height estimates, although target's sex was more effective than ratios of head to trunk. In Experiments 2 and 3, the recognizability of target persons was reduced, respectively, by inverting the person photographs and by degrading the various features of the photographs. The main results were that (1) the correlation between mean estimated height and actual height was .88 for the inverted photographs and was .80 , on average, for the degraded drawings; (2) target's sex was consistently influential in both experiments; (3) vertical ratio was effective for the inverted photographs and the mildly degraded drawings but not for the extremely degraded drawings in which only outlines of target persons were depicted; and (4) the estimation for the outlined pictures was likely to be influenced by fit of clothes to targets' bodies. From these findings, person stimuli were considered to provide a stable metric stick of visual space under naturalistic circumstances.
\end{abstract}

We are generally capable of estimating the height of a person very accurately, even if the person is viewed at a great distance from which many of the distance cues listed in textbooks are not clearly identifiable (Higashiyama \& Kitano, 1991; Leibowitz \& Harvey, 1967, 1969). Such accurate estimation could be achieved if size information is contained in the target person per se. If this inference is valid, we can also make precise height estimates for a photographed person in which cues to distance are thoroughly eliminated. The goal of this study is to demonstrate that there can be accurate height estimates for persons seen in pictures and to explore the sources of information that make such accurate estimation possible.

One possible source of accurate height estimates for persons is the familiar size of persons (see, e.g., Carlson \& Tassone, 1971; Fillembaum, Schiffman, \& Butcher, 1965; Fitzpatrick, Pasnak, \& Tyer, 1982; Gogel, 1969, 1981; Gogel \& Da Silva, 1987a, 1987b; Higashiyama, 1982, 1984; Predebon, 1992, 1994). We believe that adults are $140-190 \mathrm{~cm}$ tall. Therefore, if we recognize a target to be a person, this belief can be available for size estimation. In addition to this familiar size, recognition of the

This study was supported in part by Grants-in-Aid for Scientific Research (No. 08234103) provided by the Japanese Ministry of Education, Science, and Culture. We would like to express our thanks to $\mathrm{H}$. A. Sedwick, G. Bingham, and an anonymous reviewer for their critical reading of the earlier manuscript. Requests for reprints should be sent to K. Kato, Department of Psychology, Faculty of Letters, Nagoya University, Chigusa-ku, Nagoya 464-8601, Japan (e-mail: k46378a@nucc. cc.nagoya-u.ac.jp). sex of the target person is useful. If the target's sex is recognized by his or her body contour, hair style, and clothes, the possible height is much more narrowly settled, because we believe that men are generally taller than women. Nelson, Biernat, and Manis (1990), indeed, found a strong effect of target's sex on height estimates for photographed persons. Although they attempted to eliminate the effect of sex stereotypes (i.e., a belief that men are generally taller than women) by discouraging the subject's reliance on the target's sex or by offering cash rewards for accuracy, the sex stereotype remained effective.

Another possible source is the relative size among parts of the body. Medawar (1944), Todd, Mark, Shaw, and Pittenger (1980), Pittenger and Todd (1983), Alley (1983), and Pittenger (1990) demonstrated that, during growth, the relative size of the head to the trunk gradually decreases and, as a result, the relative size for adults becomes much smaller than that for infants; accordingly, persons with smaller heads relative to their trunks tend to look more grown and taller. However, there may be two issues concerning the relative size of the head to the trunk. The first is the question of whether the relative size is effective in discriminating heights among adults as well as between adults and infants. We did not know whether, for the adults whose growth has stopped, the relative size is still negatively correlated with their stature. The second issue is the definitions of head size and trunk size. What is exactly meant by size has not been clear. It is possible to interpret the size as vertical linear height, lateral linear width, or two-dimensional area. We explored how these variables affect height estimates. 


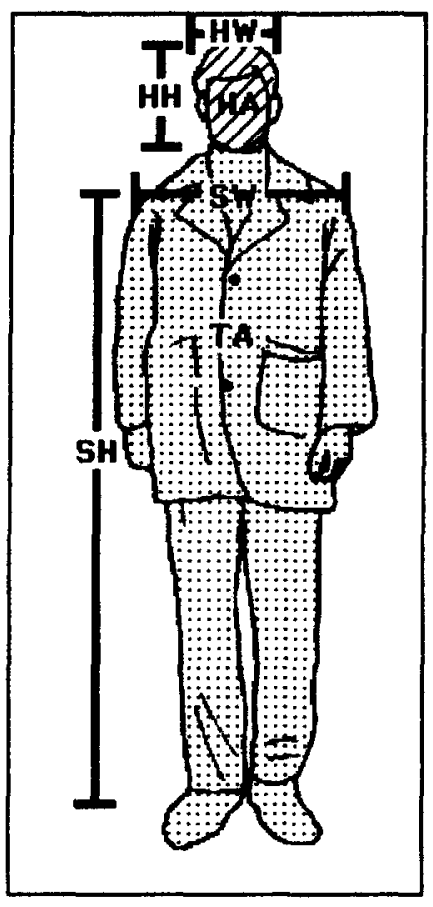

Figure 1. Definitions of body parts that may affect height estimates. HW, head width; HH, head height; SW, shoulder width; SH, shoulder height; HA (hatched portion), head area; TA (dotted portion), trunk area.

The third possible source is the fit of clothes to the body. Although there is not sufficient evidence, someone may believe that tight-fitting clothes make the body appear much slenderer than loose-fitting clothes, and a slenderer person tends to be judged to be taller. However, the relation between fit of clothes and apparent height may not be as reliable as is the relation between the target's sex and apparent height. If our estimation was based on fit of clothes, we would not achieve the accurate height estimates that prevail in natural viewing situations.

In this study, we report three experiments. In Experiment 1 , the subjects estimated the height of young adults standing erect in photographs, and we explored, with the method of multiple linear regression, the cues that might affect height estimates. In subsequent experiments, we investigated how the effective variables found in Experiment 1 interact with recognizability of target persons. The recognizability of target persons was manipulated by inverting the realistic person photographs so that they were upside down (Experiment 2 ) and by degrading the various visual features of the photographed persons (Experiment 3 ). We sought the robust variables influencing height estimates, even with such poor picture information.

\section{EXPERIMENT 1}

\section{Method}

Subjects. Thirty undergraduates rated the height of photographed persons. Half the subjects were men, and the rest were women. None had ever seen any target person in pictures. An additional 10 undergraduates ( 5 men and 5 women) rated the fit of the clothes that the photographed persons wore.

Stimuli. Fifty colored photographs of Japanese people ( 26 men and 24 women, $18-28$ years old) were used as targets. Their real statures, which would be obtained if the target persons took off their shoes, ranged from 143 to $186 \mathrm{~cm}$. We did not have any complex criteria for selecting the target persons. The only explicit criterion used was that the young adults selected should have a wide distribution of statures. The target persons wore any clothes and shoes they might like.

To eliminate conventional cues to size and distance, each target person stood in front of a textureless homogeneous white wall. A frontal view of the person was then taken with a Pentax Espio 110 autofocus camera (Asahi Optics Co.), with a focal distance of $38-110 \mathrm{~mm}$. The camera was so placed that the height of the person equaled $107 \mathrm{~mm}$ in a picture frame that was $117 \mathrm{~mm}$ high $\times$ $82 \mathrm{~mm}$ wide. The camera was adjusted to the height of the waist of the target person. The pictures were taken outdoors on sunny days in February. The sex of each photographed person was very easily recognized at a glance.

For analyzing the relation between body parts and estimated height, we measured the length and area of the following body parts in pictures: the head width, shoulder width, head height, shoulder height, head area, and trunk area. Figure 1 shows the graphic definitions of these variables. The widths and heights were directly obtained with a measuring rule to an accuracy of $1 \mathrm{~mm}$. Head and trunk areas were measured in the number of pixels, by making use of NIH IMAGE/68k 1.58b11 software after each picture was taken in a Power Macintosh 8500/120 computer connected with an Epson GT- 8500 scanner. The ratio of head to trunk was obtained by dividing the head area by the trunk area. We did not measure leg length and waist height, because 22 target persons ( 6 men and 16 women) wore skirts and/or overcoats that occluded both the crotch and the waist.

Procedure. There were two groups of subjects. One group of 30 subjects judged the height of the target persons in pictures, whereas another group of 10 subjects judged the fit of clothes of the target persons. The same 50 target pictures were used for both groups of subjects.

For the height rating, the subjects were told to judge each target's real stature that would be obtained if the target person took off his or her shoes. After shuffling the 50 pictures, the experimenter handed over the pile of pictures to each subject. For a given trial, the subject observed a person in a picture and rated his or her height in terms of a 5 -point scale, with $1=$ under $150 \mathrm{~cm}, 2=150 \mathrm{~cm}$ or more but under $160 \mathrm{~cm}, 3=160 \mathrm{~cm}$ or more but under $170 \mathrm{~cm}, 4=170 \mathrm{~cm}$ or more but under $180 \mathrm{~cm}$, and $5=180 \mathrm{~cm}$ or more. The subject wrote his or her response on a sheet of paper. After that, the subject turned over the picture and observed another picture for the next trial. Accordingly, the subjects could not make a simultaneous visual comparison between target persons.

For the fit rating, the subjects judged the fit of the clothes of the photographed persons. In the same way as for the height rating, the experimenter handed over the pile of 50 pictures that were adequately shuffled. Each subject observed one target person at a time and rated the fit of clothes in terms of a 5-point scale, with $1=$ very tight, $2=$ tight, $3=$ neutral, $4=$ loose, and $5=$ veryloose. The mean fit for each target person was used as a predictor for analysis.

In both height and fit ratings, any correction of responses was not allowed. The subjects worked at their own pace; no limitation on the time needed for rating was imposed on the subject. The experimenter ran 2 or 3 subjects at once.

\section{Results and Discussion}

Figure 2 shows the mean rating of all subjects against the real full height of the target persons. For all the target persons, the Pearson coefficient of correlation between 


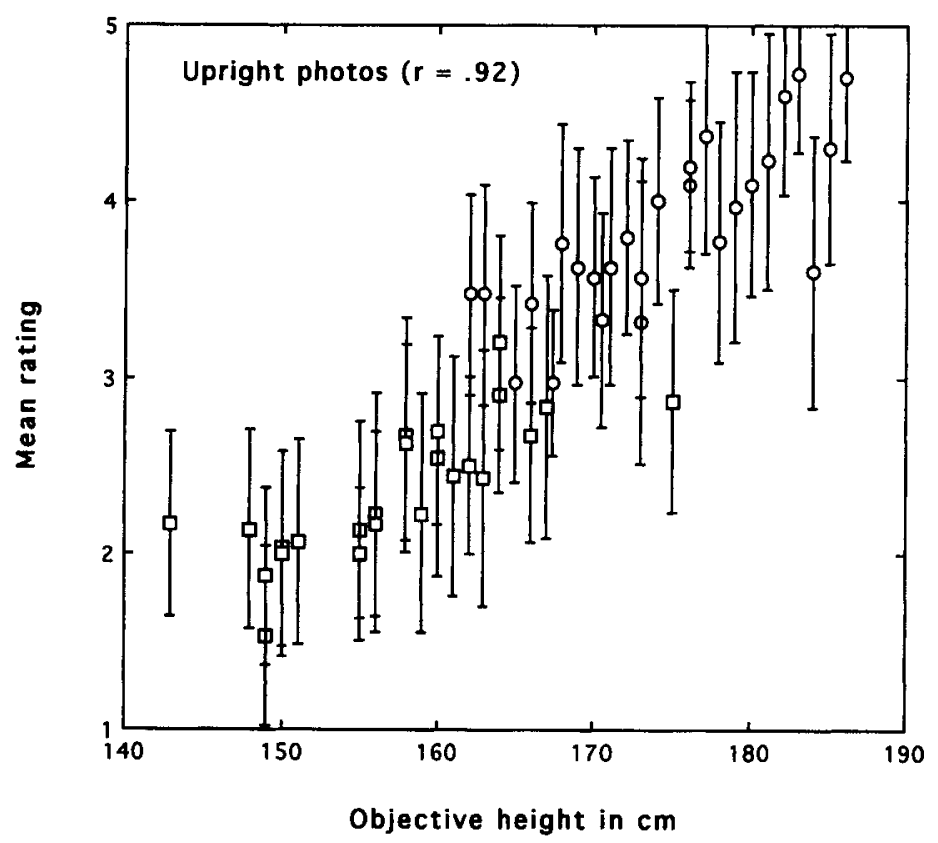

Figure 2. Mean height judgments for the photographed upright persons as a function of actual height. Circles represent men, and squares represent women. The vertical bars attached to the marks represent standard deviations.

mean rating and real height was .92 , which was significantly different from zero $(p<.001, n=50)$. The correlation for male pictures was $.82(p<.001, n=26)$, and the correlation for female pictures was $.80(p<.001, n=24)$. A correlation was also obtained from the ratings of each subject. The obtained individual correlations ranged from .60 to .87 and averaged $.77(S D=.06)$. Thus, it is suggested that, since the pictures did not include conventional cues as to size and distance, the accurate height ratings were based on size information involved in target persons per se.

By stepwise performing multiple linear regression analyses, we attempted to find the predictors that influenced the height ratings for target persons. The variables to be considered were target's sex, the ratio of head area to trunk area (area ratio), the ratio of head width to shoulder width (horizontal ratio), the ratio of head height to stature (vertical ratio), and the mean fit of clothes (fit) for each target. Table 1 shows the mean, $S D$, minimum, and maximum of the full body height, area ratio, horizontal ratio, vertical ratio, and fit of clothes. Since target's sex is a nominal scale, a male was given a score of 0 and a female a score of 1 . The strategy in these analyses was to determine as few predictors with a high squared multiple correlation $R^{2}$ as possible.

Table 2 shows the results. We first used the five variables as predictors. The obtained $R^{2}$ was .862 , and the $\beta$ weights for target's sex $[-.611, t(44)=7.79, p<.001]$, horizontal ratio $[-.157, t(44)=2.33, p<.05]$, and vertical ratio $[-.263, t(44)=4.15, p<.001]$ were significant. Since the $\beta$ for target's sex was overwhelmingly larger than that for any other predictor, target's sex was included as a predictor in any model of the subsequent analyses.

For the analyses using four variables, the predictors were target's sex and three variables that were systematically selected from area ratio, horizontal ratio, vertical ratio, and fit. The highest $R^{2}$ we obtained from the four possible models was .857 , and the $\beta$ s for sex $[-.660$, $t(45)=9.74, p<.001]$, horizontal ratio $[-.175, t(45)=$ $2.68, p<.05]$, and vertical ratio $[-.286, t(45)=4.74$, $p<.001]$ were significant.

For the analyses using three variables, the predictors were target's sex and two variables that were systematically selected from area ratio, horizontal ratio, vertical ratio, and fit. The highest $R^{2}$ we obtained from the six possible models was .849 , and the $\beta$ s for sex $[-.661$, $t(46)=9.59, p<.001]$, horizontal ratio $[-.192, t(46)=$ $2.89, p<.01]$, and vertical ratio $[-.294, t(46)=4.80, p<$ $.001]$ were significant.

Table 1

Mean, Standard Deviation, Minimum, and Maximum of the Real Full Body Height (in Centimeters), Area Ratio, Vertical Ratio, Horizontal Ratio, and Fit of Clothes for the 50 Target Persons

\begin{tabular}{lcccc}
\hline \multicolumn{1}{c}{ Variable } & $M$ & $S D$ & Minimum & Maximum \\
\hline Body height & 166.2 & 11.0 & 143.0 & 186.0 \\
Area ratio & .078 & .017 & .056 & .115 \\
Vertical ratio & .154 & .010 & .131 & .179 \\
Horizontal ratio & .462 & .057 & .375 & .633 \\
Fit & 3.03 & 0.63 & 1.40 & 4.40 \\
\hline
\end{tabular}


Table 2

Squared Multiple Regression Coefficient $\left(R^{2}\right)$ and $\beta$ Weights Obtained for the Height Estimates of the Upright Photographs

\begin{tabular}{ccccccc} 
& & \multicolumn{5}{c}{ Predictors } \\
\cline { 2 - 6 } NP & $R^{2}$ & Sex & Area & Hor. & Ver. & Fit \\
\hline 5 & .862 & $-.611 \ddagger$ & -.099 & $-.157^{*}$ & $-.263 \ddagger$ & -.090 \\
4 & .857 & $-.660 \ddagger$ & & $-.175^{*}$ & $-.286 \ddagger$ & -.092 \\
4 & .845 & $-.661 \ddagger$ & -.141 & & $-.261 \ddagger$ & -.110 \\
4 & .808 & $-.632 \ddagger$ & $-.204^{*}$ & -.154 & & -.106 \\
4 & .854 & $-.610 \ddagger$ & -.103 & $-.173^{*}$ & $-.269 \ddagger$ & \\
3 & .791 & $-.680 \ddagger$ & $-.244 \dagger$ & & & -.125 \\
3 & .849 & $-.661 \ddagger$ & & -.192 & $-.294 \ddagger$ & \\
3 & .797 & $-.631 \ddagger$ & $-.212^{*}$ & $-.173^{*}$ & & \\
3 & .833 & $-.665 \ddagger$ & -.151 & & $-.269 \ddagger$ & \\
3 & .786 & $-.747 \ddagger$ & & $-.194^{*}$ & & -.114 \\
3 & .835 & $-.742 \ddagger$ & & & $-.296 \ddagger$ & -.117 \\
\hline
\end{tabular}

Note-NP, number of predictors; Hor., horizontal ratio; Ver., vertical ratio. Significance of each predictor was evaluated with $t$ tests $(d f=50-$ NP - 1). $\quad{ }^{*} p<.05 . \quad{ }^{\dagger} p<.01 . \quad \ddagger p<.001$

The finding that the $\beta$ for target's sex was significant in any model shown in Table 2 suggested that the sex stereotype about height reduces the possible range of the height for target persons. If the target person was identified as being male, his stature tended to be estimated to be higher than $160 \mathrm{~cm}$. If the target person was identified as being female, her stature tended to be estimated to be between 150 and $170 \mathrm{~cm}$. This result parallels the Nelson et al. (1990) study.

From Table 2, several body proportions were apparently effective in height estimates, although they were less effective than target's sex. In particular, the $\beta$ s for vertical and horizontal ratios were consistently significant. This means that the more the vertical (or horizontal) ratio, the smaller the mean rating. In contrast, area ratio and fit of clothes were ineffective in almost all models.

The correlation between mean rating and real height was also high for male pictures alone $(r=.82)$ and for female pictures alone $(r=.80)$. From this finding, one may consider that, even if sex is removed from the set of predictors, horizontal and vertical ratios are effective in height ratings. We examined this prediction by performing multiple linear regression analyses on the ratings for the single-sex targets. The predictors were the four or three variables that were systematically chosen from area ratio, horizontal ratio, vertical ratio, and fit.

Table 3 shows the results. With the four-variable model that was applied to male pictures, we obtained $R^{2}=.548$, and the $\beta$ s for horizontal ratio $[-.409, t(21)=2.49, p<$ $.05]$ and vertical ratio $[-.416, t(21)=2.13, p<.05]$ were significant; for the analyses using three variables, the best-fitting function $\left(R^{2}=.543\right)$ that we obtained from the four possible models indicated that the $\beta$ s for horizontal ratio $[-.446, t(22)=3.07, p<.01]$ and vertical ratio $[-.480, t(22)=3.28, p<.01]$ were significant. Similarly, with the four-variable model that was applied to female pictures, we obtained $R^{2}=.559$, and the $\beta$ for vertical ratio $[-.582, t(19)=3.66, p<.01]$ was significant; for the analyses using three variables, the best-fitting function $\left(R^{2}=.555\right)$ that we obtained from the four possible models indicated that the $\beta$ for vertical ratio $[-.581$, $t(20)=3.73, p<.01]$ was significant. Thus, vertical and horizontal ratios were effective in the analyses of singlesex targets.

For comparison with the $\beta$ s in Table 2, we also performed multiple linear regression analyses on real body heights of the target persons, using the same five predictors as in the analyses of height judgments. Table 4 clearly indicates that target's sex and vertical ratio were very good predictors of real height, because these $\beta$ s were significant in all models. However, the $\beta$ s for area ratio were significant in some models but not in others, and the $\beta$ s for horizontal ratio and fit were not significant in any model.

Interestingly, target's sex and vertical ratio were consistently significant in predicting both judged and real heights. This similarity of significant $\beta$ s implies that the subjects responded to the ecologically valid variables that were directly related to real height, although they might not have been aware of what variables were useful.

\section{EXPERIMENT 2}

In Experiment 2, the subjects observed the upsidedown inverted photographs of persons. The goal of Experiment 2 was to examine whether accurate height estimates would be maintained for the inverted person

Table 3

Squared Multiple Regression Coefficient $\left(R^{2}\right)$ and

$\beta$ Weights Obtained for the Height Estimates of the Single-Sex, Upright Photographed Persons

\begin{tabular}{|c|c|c|c|c|c|c|c|c|c|c|}
\hline \multirow[b]{2}{*}{ NP } & \multicolumn{5}{|c|}{ Male } & \multicolumn{5}{|c|}{ Female } \\
\hline & $R^{2}$ & Area & Hor. & Ver. & Fit & $R^{2}$ & Area & Hor. & Ver. & Fit \\
\hline 4 & $.548^{\dagger}$ & -.104 & $-.409^{*}$ & $-.416^{*}$ & -.296 & $.559 \dagger$ & -.202 & -.207 & $-.582 \dagger$ & -.067 \\
\hline 3 & $.543 \dagger$ & & $-.446 \dagger$ & $-.480 \dagger$ & -.295 & $.523 \dagger$ & & -.243 & $-.628+$ & -.076 \\
\hline 3 & $.415 \dagger$ & -.324 & & -.247 & -.327 & $.521 \dagger$ & -.239 & & $-.606 \dagger$ & -.107 \\
\hline 3 & $.451 \dagger$ & $-.383^{*}$ & -.288 & & $-.332^{*}$ & .249 & -.338 & -.278 & & -.060 \\
\hline 3 & $.463 \div$ & -.102 & $-.437^{*}$ & $-.461^{*}$ & & $.555 \dagger$ & -.205 & -.221 & $-.581 \dagger$ & \\
\hline
\end{tabular}

Note-NP, number of predictors; Hor., horizontal ratio; Ver., vertical ratio. Significance of each predictor was evaluated with $t$ tests: For the male targets, $d f=26-\mathrm{NP}-1$; for the female targets, $d f=24-\mathrm{NP}-1 .{ }^{*} p<.05 . \quad{ }^{\dagger} p<.01 . \quad{ }^{\dagger} p<.001$. 
Table 4

Squared Multiple Regression Coefficient $\left(R^{2}\right)$ and $\beta$ Weights Obtained for the Real Heights of the Target Persons

\begin{tabular}{ccccccc}
\hline & & \multicolumn{5}{c}{ Predictors } \\
\cline { 2 - 7 } NP & $R^{2}$ & Sex & Area & Hor. & Ver. & Fit \\
\hline 5 & .749 & $-.49 \dagger^{\dagger}$ & -.117 & -.156 & $-.330 \dagger$ & -.053 \\
4 & .742 & $-.548 \dagger$ & & -.177 & $-.358 \dagger$ & -.055 \\
4 & .732 & $-.539 \dagger$ & -.158 & & $-.328 \dagger$ & -.073 \\
4 & .663 & $-.516^{\dagger}$ & $-.249^{*}$ & -.152 & & -.072 \\
4 & .746 & $-.48 \dagger^{\dagger}$ & -.120 & -.165 & $-.333 \dagger$ & \\
3 & .648 & $-.563 \dagger$ & $-.289^{*}$ & & & -.092 \\
3 & .739 & $-.548 \dagger$ & & -.187 & $-.362 \dagger$ & \\
3 & .658 & $-.515 \dagger$ & $-.255^{*}$ & -.164 & & \\
3 & .727 & $-.542 \dagger$ & -.165 & & $-.333 \dagger$ & \\
3 & .631 & $-.656^{\dagger}$ & & -.200 & & -.082 \\
3 & .720 & $-.630^{\dagger}$ & & & $-.368 \dagger$ & -.080 \\
\hline
\end{tabular}

Note-NP, number of predictors; Hor., horizontal ratio; Ver., vertical ratio. Significance of each predictor was evaluated with $t$ tests $(d f=50$ $-\mathrm{NP}-1) . \quad{ }^{*} p<.05 . \quad{ }^{+} p<.001$.

images and whether target's sex and the relation of head to trunk would be cues to height estimation.

An inversion of retinal image yields drastic changes in figure (Howard, 1982; Köhler, 1940), face and word (Rock, 1973), and size and distance perception (Higashiyama, 1996; Rock \& Kaufman, 1962). Why does the inversion of retinal image yield such perceptual changes? Although there are several possible explanations, Rock (1988) assumed that, in viewing an inverted face, it is difficult to cope with the relation of parts, such as a mouth, a nose, and eyes. If Rock's (1988) assumption is applic- able to a human body in which a head, a trunk, and limbs should be considered as parts, the relation of head to trunk would not adequately be coped with in the inverted photographs. This suggests that the ratio of head size to trunk size in the inverted picture images may be less effective on height estimates. However, accurate height estimates would be maintained, because recognition of target's sex would not be damaged by inverting the retinal image of a human body.

\section{Method}

Subjects. Twenty-two undergraduates were newly recruited for Experiment 2. Half the subjects were men, and the rest were women. None of them had ever seen the persons in the pictures.

Stimuli. The same 50 photographs as those used in Experiment 1 were used as stimuli.

Procedure. Each subject generally followed the same procedure as that for the height rating in Experiment 1, with one exception. The subject indicated the height of inverted person images with verbal estimates expressed in centimeters rather than with the 5-point scale. It was emphasized in the instructions that the subjects should judge the actual height of real persons (i.e., objective instructions) and could use any numbers they liked, including decimals and fractions.

\section{Results and Discussion}

Figure 3 shows the mean estimates of all subjects against the real full height of the target persons. The correlation for all target persons was $.88(p<.001, n=50)$. The correlation for male targets was .79 $(p<.001, n=$ $26)$, and the correlation for female targets was .64 ( $p<$ $.001, n=24)$. The individual correlations ranged from .66 to .87 and averaged $.77(S D=.06)$.

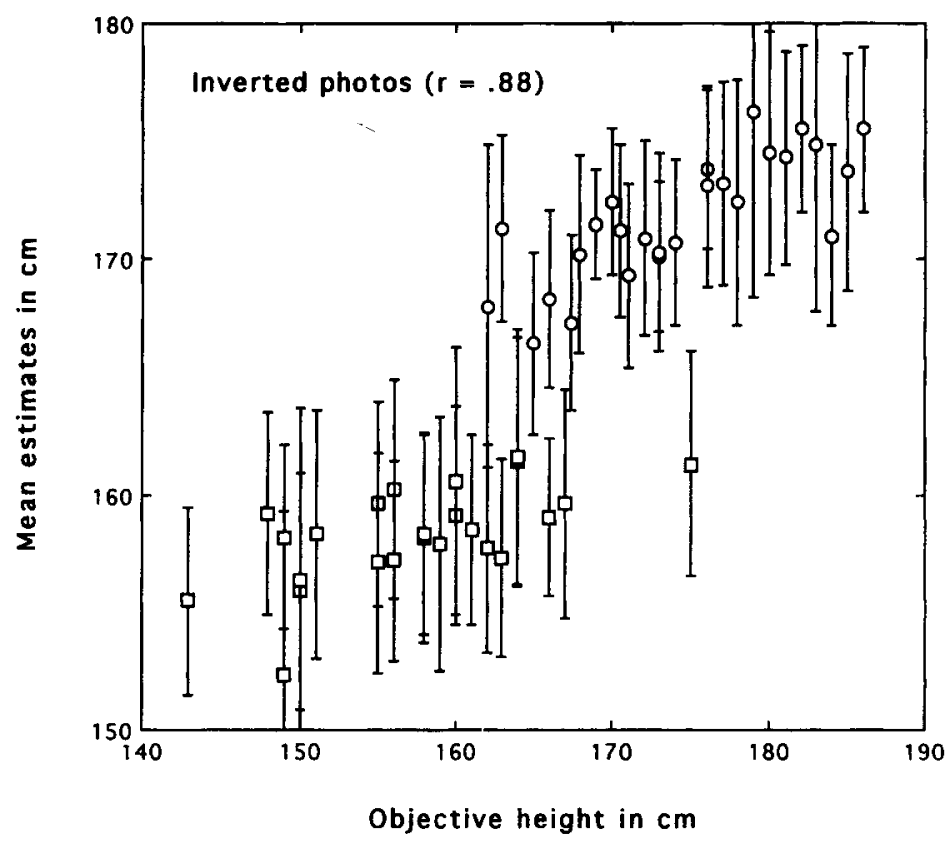

Figure 3. Mean height judgments for the photographed inverted persons as a function of actual height. Circles represent men, and squares represent women. The vertical bars attached to the marks represent standard deviations. 
Table 5

Squared Multiple Regression Coefficient $\left(R^{2}\right)$ and $\beta$ Weights Obtained for the Height Estimates of the Inverted Photographs

\begin{tabular}{ccccccc}
\hline & & \multicolumn{5}{c}{ Predictors } \\
\cline { 3 - 7 } NP & $R^{2}$ & Sex & Area & Hor. & Ver. & Fit \\
\hline 5 & .921 & $-.833 \ddagger$ & -.052 & -.045 & $-.125^{*}$ & -.079 \\
4 & .920 & $-.858 \ddagger$ & & -.054 & $-.137 \dagger$ & -.080 \\
4 & .920 & $-.847 \ddagger$ & -.063 & & $-.124^{*}$ & -.085 \\
4 & .909 & $-.843 \ddagger$ & -.102 & -.044 & & -.087 \\
4 & .915 & $-.832 \ddagger$ & -.055 & -.059 & $-.130^{*}$ & \\
3 & .907 & $-.857 \ddagger$ & -.113 & & & $-.092^{*}$ \\
3 & .914 & $-.859 \ddagger$ & & -.069 & $-.144 \dagger$ & \\
3 & .902 & $-.842 \ddagger$ & -.108 & -.059 & & \\
3 & .913 & $-.851 \ddagger$ & -.071 & & $-.130^{*}$ & \\
3 & .903 & $-.900 \ddagger$ & & -.063 & & -.091 \\
3 & .918 & $-.884 \ddagger$ & & & $-.140 \dagger$ & -.088 \\
\hline
\end{tabular}

Note-NP, number of predictors; Hor., horizontal ratio; Ver., vertical ratio. Significance of each predictor was evaluated with $t$ tests $(d f=50-$ $\mathrm{NP}-1) . \quad{ }^{*} p<.05 . \quad{ }^{\dagger} p<.01 . \quad{ }^{\ddagger} p<.001$.

Note that, for the inverted picture images, we still obtained a high correlation (.88) between mean estimates and real heights, although this correlation was not as high as the correlation for the upright photographs (.92). In addition, the averaged individual correlation was compatible with that in Experiment 1 (.77). These results suggest that accurate height estimates were maintained for the inverted picture images.

For comparison with the results of Experiment 1, multiple regression analyses were performed with the same models as those used in Experiment 1. Table 5 shows the results. When the five variables were used, the obtained $R^{2}$ was .921 , and the $\beta$ s for sex $[-.833, t(44)=14.05, p<$ $.001]$ and vertical ratio $[-.125, t(44)=2.60, p<.05]$ were significant.

For the analyses using four variables, the highest $R^{2}$ we obtained from the four possible models was .920 , which was accidentally obtained for the two models: In one model, in which the predictors were sex, horizontal ratio, vertical ratio, and fit, the $\beta$ s for sex $[-.858, t(45)=$ $16.90, p<.001]$ and vertical ratio $[-.137, t(45)=3.02$, $p<.01]$ were significant; in another model, in which the predictors were sex, area ratio, vertical ratio, and fit, the $\beta$ s for sex $[-.847, t(45)=14.88, p<.001]$ and vertical ratio $[-.124, t(45)=2.60, p<.05]$ were significant.

For the analyses using three variables, the highest $R^{2}$ we obtained from the six possible models was .918 , and the $\beta$ s for sex $[-.884, t(46)=19.50, p<.001]$ and vertical ratio $[-.140, t(46)=2.05, p<.01]$ in this model were significant. These regression analyses generally indicated that target's sex and vertical ratio were major determinants of height estimates.

The effects of target's sex were enhanced in Experiment 2: The mean $\beta$ for target's sex, which was taken across the 11 models in Table 5, was -.855 , whereas the corresponding mean $\beta$ in Experiment 1 was -.664. In Figure 3 , we indeed recognize two clusters of data points that depended on target's sex.

Body proportions were less effective in Experiment 2 than in Experiment 1 . First, only vertical ratio was effective in Experiment 2, although both horizontal and vertical ratios were effective in Experiment 1 . Second, the mean $\beta$ for the vertical ratio $(-.133)$ in Experiment 2 was smaller than the corresponding mean $\beta$ in Experiment $1(-.277)$. To illustrate the contribution of vertical ratio in each experiment, the relations between mean judgment and vertical ratio were shown in Figure 4, where the simple correlation for the upright photographs was higher than that for the inverted photographs.

It seems that the results of Experiment 2 supported the extension of Rock's (1988) assumption: By inverting picture images, it may be difficult to cope with the relation of head to trunk, although the sizes of the head and the trunk by themselves are adequately perceived. It is probable that, since it was difficult to use the body proportions in the inverted picture images, the subjects strongly relied on target's sex in height estimation. This is the rea-
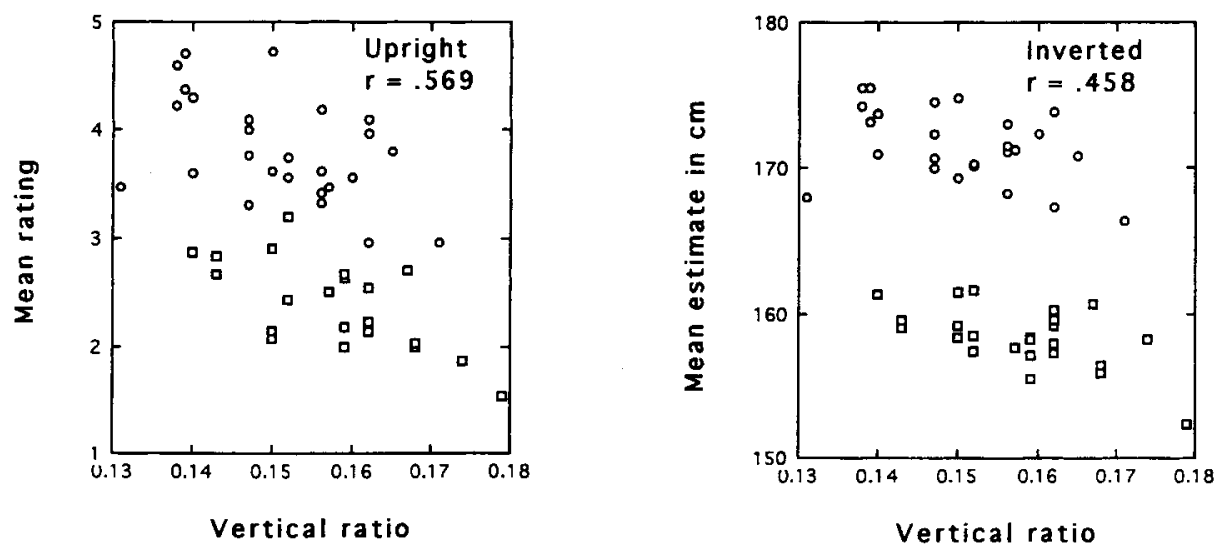

Figure 4. Mean height judgments for (left panel) upright and (right panel) inverted persons as a function of vertical ratio (i.e., ratio of head height to full body height). Circles represent men, and squares represent women. 

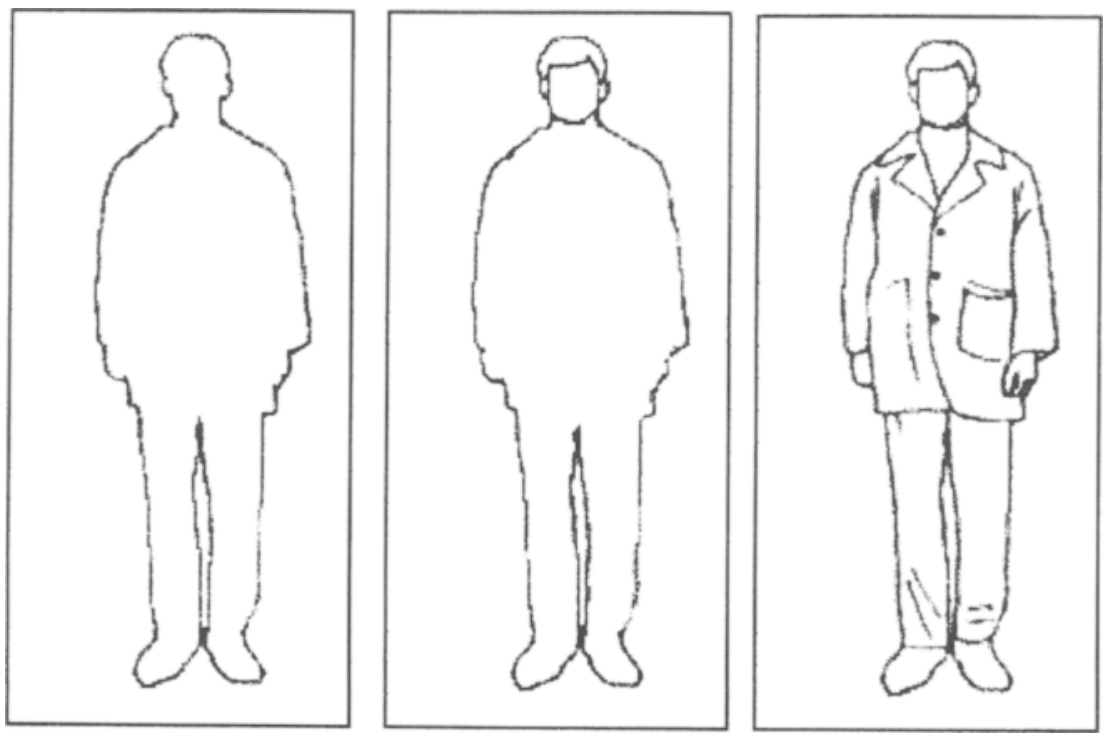

Figure 5. Examples of the silhouette, face drawings, and clothes drawings.

son that the $\beta$ for target's sex was enhanced in this experiment.

\section{EXPERIMENT 3}

An implication of the results of Experiments 1 and 2 was that the effects of target's sex on height estimates were strong and consistent but the effects of body proportions were not as consistent as target's sex. We assumed that these unstable effects of body proportions are due to low visual recognizability that may take place by changing the orientation of the target images. To examine this assumption further, the subjects in Experiment 3 estimated the height of persons in three sets of pictures that were obtained by tracing the photographed persons partially. It was assumed that, for the visually degraded drawings of persons, target's sex would still be effective but body proportions would not.

\section{Method}

Subjects. Seventy undergraduates who had never served in Experiments 1 and 2 were newly recruited for Experiment 3 . Half the subjects were men, and the rest were women.

Stimuli. The stimuli were made from the photographs used in Experiment 1. Each stimulus was depicted on a piece of white paper ( $13 \mathrm{~cm}$ high $\times 6 \mathrm{~cm}$ wide) by tracing the photographed person with black ink. There were three degraded drawings for each photographed person. Examples of these drawings are shown in Figure 5. In the silhouette condition, only the outlines of each photographed person were traced. In the face condition, the face contours and hairlines of each photographed person were superimposed on the silhouette drawing, so that the head was easily discriminated from the trunk. In the clothes condition, the decoration and wrinkle of the clothes of each photographed person were superimposed on the face drawings. In any condition, facial features, such as a mouth, a nose, and eyes, were not drawn. Thus, a total of 150 drawings, 50 for each degradation, were used as stimuli.

Procedure. Sixty subjects were randomly assigned to one of the three degradations, with the restriction that 10 men and 10 women were involved in one group. The subjects followed the same procedure as that in Experiment 2. The order of the 50 drawings for each degradation was randomly determined for each subject. Each subject observed one drawing at a time and indicated the height of the depicted person with verbal estimates expressed in centimeters. The subjects were to judge the actual height of real persons and could use any numbers they liked, including decimals and fractions.

The additional 10 subjects were asked to identify the sex of the 150 depicted persons. The trials consisted of three blocks. To avoid prior knowledge of sex that might be obtained by seeing the detailed drawings in earlier blocks, each subject first judged the silhouette drawings, next the face drawings, and lastly the clothes drawings. The order of the 50 drawings for each degradaiion was randomly determined for each subject.

\section{Results and Discussion}

For the silhouette, face, and clothes drawings, the mean rates of correct identification of target's sex were $.91, .91$, and .95 , respectively, and for male and female drawings, they were .96 and .89 , respectively. Therefore, the sex of the depicted persons was well identified, on the whole.

A two-way analysis of variance (ANOVA) with repeated measures on the inverse-sine transformed values of the correct rates indicated that the main effects of drawing $[F(2,18)=6.28, p<.01]$ and target's sex $[F(1,9)=5.91$, $p<.05]$ were significant and that the interaction of drawing and target's sex was significant $[F(2,18)=3.91$, $p<.05]$. The correct rate for female drawings was lower in the silhouette (.85) and face (.86) drawings than in the clothes drawings (.95), whereas the correct rates for male drawings were much the same in the silhouette (.97), 

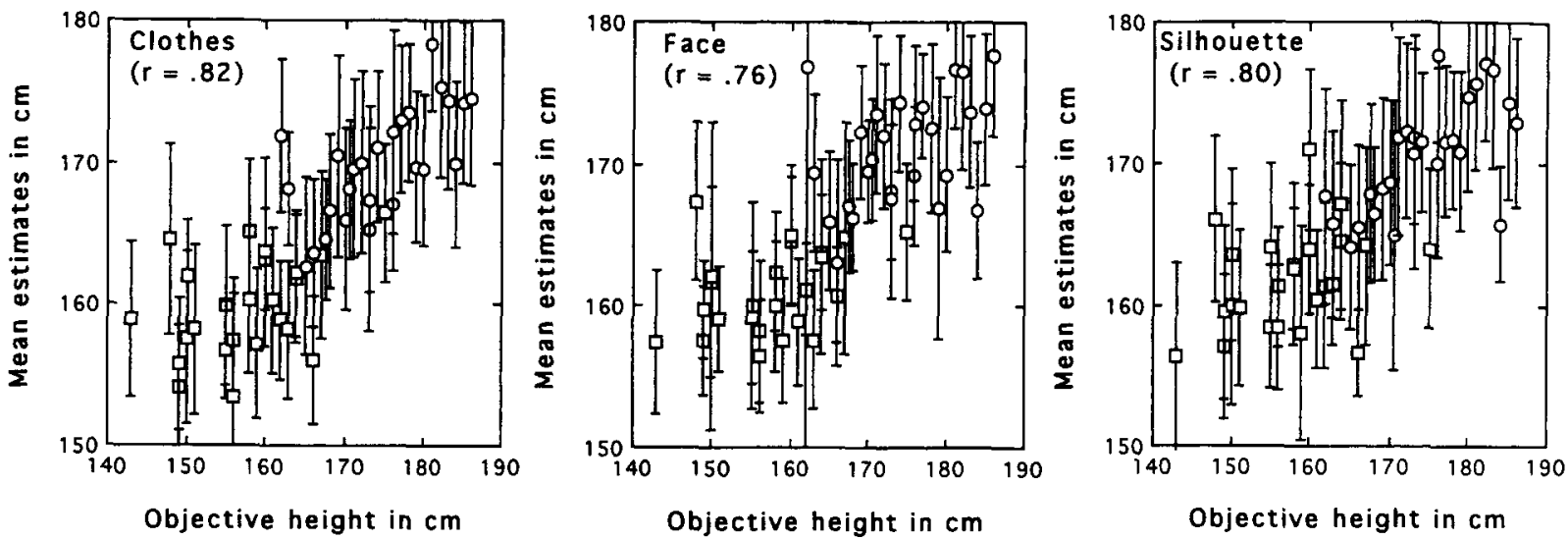

Figure 6. Mean height judgments for the depicted persons (i.e., clothes, face, and silhouette) as a function of actual height. Circles represent men, and squares represent women. The vertical bars attached to the marks represent standard deviations.

face (.96), and clothes (.96) drawings. Thus, it was suggested that correct identification of the female targets was difficult with extremely degraded drawings.

Figure 6 shows the relations between mean estimates of all subjects and real full height of the target persons. For any condition, the mean estimates were strongly related to real height (for the silhouette condition, $r=.80$, $p<.001, n=50$; for the face condition, $r=.77, p<.001$, $n=50$; for the clothes condition, $r=.82, p<.001, n=$ 50 ). Correlations for male drawings were significant in all conditions (for the silhouette condition, $r=.69, p<$ $.001, n=26$; for the face condition, $r=.40, p<.05, n=$ 26 ; for the clothes condition, $r=.67, p<.001, n=26$ ), but correlations for female drawings were not significant (for the silhouette condition, $r=.34$; for the face condition, $r=.35$; for the clothes condition, $r=.39$ ).

Individual correlations between height estimates and real height were obtained for each degradation. For the silhouette, face, and clothes conditions, the averaged individual correlations were $.59(S D=.16), .60(S D=.16)$, and $.67(S D=.12)$, respectively. The differences among these correlations were not significant, but the mean correlation (.62) was significantly different from the averaged individual correlations in Experiments $1[t(88)=5.35, p<$ $.001]$ and $2[t(80)=4.58, p<.001]$. Accordingly, it was suggested that the height estimates for the degraded drawings were less accurate than those for the realistic photographs.

For comparison with the results of Experiments 1 and 2 , we performed multiple regression analyses with the predictors of target's sex, area ratio, horizontal ratio, vertical ratio, and fit. Table 6 shows the results of the silhouette condition. For the analysis using the five variables, the obtained $R^{2}$ was .701 , and the $\beta$ s for sex [ -.510 , $t(44)=4.42, p<.001]$ and fit $[-.188, t(44)=2.22, p<$ $.05]$ were significant. For the analyses using four variables, the highest $R^{2}$ we obtained from the four possible models was .698, and the $\beta$ s for sex $[-.530, t(45)=4.80$, $p<.001]$, area ratio $[-.256, t(45)=2.20, p<.05]$, and fit $[-.195, t(45)=2.35, p<.05]$ were significant. For the analyses using three variables, the highest $R^{2}$ we obtained from the six possible models was .687 , and the $\beta \mathrm{s}$ for $\operatorname{sex}[-.538, t(46)=4.86, p<.001]$, area ratio $[-.303$, $t(46)=2.73, p<.01]$, and fit $[-.202, t(46)=2.42, p<$ $.05]$ were significant. These results suggested that target's sex and fit were consistently effective, regardless of the number of predictors, and that target's sex was more effective than fit. In contrast to the results of Experiments 1 and 2, effects of vertical ratio and horizontal ratio were not found at all; instead, area ratio was effective in several models.

Table 7 shows the results of the face condition. For the analysis using the five variables, the obtained $R^{2}$ was .786 , and the $\beta$ s for sex $[-.614, t(44)=6.29, p<.001]$ and vertical ratio $[-.275, t(44)=3.49, p<.01]$ were significant. For the analyses using four variables, the highest $R^{2}$ we obtained from the four possible models was .786, and the $\beta$ s for sex $[-.619, t(45)=6.66, p<.001]$ and vertical ratio $[-.275, t(45)=3.53, p<.01]$ were significant. For the analyses using three variables, the highest $R^{2}$ we ob-

\section{Table 6}

Squared Multiple Regression Coefficient $\left(\boldsymbol{R}^{2}\right)$ and $\beta$ Weights Obtained for the Height Estimates of the Silhouette Drawings

\begin{tabular}{ccccccc}
\hline & & \multicolumn{5}{c}{ Predictors } \\
\cline { 3 - 7 } $\mathrm{NP}$ & $R^{2}$ & Sex & Area & Hor. & Ver. & Fit \\
\hline 5 & .701 & $-.510 \ddagger$ & -.240 & -.062 & -.119 & $-.188^{*}$ \\
4 & .674 & $-.628 \ddagger$ & & -.105 & -.176 & $-.193^{*}$ \\
4 & .698 & $-.530 \ddagger$ & $-.256^{*}$ & & -.118 & $-.195^{*}$ \\
4 & .690 & $-.519 \ddagger$ & $-.287^{*}$ & -.060 & & $-.195^{*}$ \\
4 & .668 & $-.507 \ddagger$ & -.248 & -.095 & -.132 & \\
3 & .687 & $-.538 \ddagger$ & $-.303 \dagger$ & & & $-.202^{*}$ \\
3 & .639 & $-.629 \ddagger$ & & -.141 & $-.192^{*}$ & \\
3 & .654 & $-.518 \ddagger$ & $-.302^{*}$ & -.095 & & \\
3 & .661 & $-.538 \ddagger$ & $-.274^{*}$ & & -.132 & \\
3 & .647 & $-.682 \ddagger$ & & -.117 & & $-.206^{*}$ \\
3 & .666 & $-.677 \ddagger$ & & & -.182 & $-.208^{*}$ \\
\hline
\end{tabular}

Note-NP, number of predictors; Hor., horizontal ratio; Ver., vertical ratio. Significance of each predictor was evaluated with $t$ tests $(d f=50-$ $\mathrm{NP}-1) . \quad{ }^{*} p<.05 . \quad{ }^{\ddagger} p<.01 . \quad{ }^{\ddagger} p<.001$. 
Table 7

Squared Multiple Regression Coefficient $\left(R^{2}\right)$ and $\beta$ Weights Obtained for the Height Estimates of the Face Drawings

\begin{tabular}{ccccccc}
\hline & & \multicolumn{5}{c}{ Predictors } \\
\cline { 3 - 7 } NP & $R^{2}$ & Sex & Area & Hor. & Ver. & Fit \\
\hline 5 & .786 & $-.614 \ddagger$ & -.145 & -.061 & $-.275 \dagger$ & -.072 \\
4 & .776 & $-.685 \ddagger$ & & -.042 & $-.310 \ddagger$ & -.075 \\
4 & .786 & $-.619 \ddagger$ & -.149 & & $-.275 \dagger$ & -.074 \\
4 & .726 & $-.636 \ddagger$ & $-.255^{*}$ & -.031 & & -.088 \\
4 & .781 & $-.613 \ddagger$ & -.148 & -.029 & $-.280 \dagger$ & \\
3 & .726 & $-.640 \ddagger$ & $-.258^{*}$ & & & -.090 \\
3 & .771 & $-.686_{\ddagger}^{\ddagger}$ & & -.056 & $-.316 \ddagger$ & \\
3 & .719 & $-.635 \ddagger$ & $-.261^{*}$ & -.028 & & \\
3 & .780 & $-.622 \ddagger$ & -.156 & & $-.280 \dagger$ & \\
3 & .693 & $-.779 \ddagger$ & & -.063 & & -.098 \\
3 & .775 & $-.705_{\ddagger}^{\ddagger}$ & & & $-.312_{\ddagger}^{\ddagger}$ & -.081 \\
\hline
\end{tabular}

Note-NP, number of predictors; Hor., horizontal ratio; Ver., vertical ratio. Significance of each predictor was evaluated with $t$ tests $(d f=50-$ $\mathrm{NP}-1) . \quad{ }^{*} p<.05 . \quad{ }^{\dagger} p<.01 . \quad{ }^{\ddagger} p<.001$.

tained from the six possible models was .780 , and the $\beta \mathrm{s}$ for sex $[-.622, t(46)=6.69, p<.001]$ and vertical ratio $[-.280, t(46)=3.60, p<.01]$ were significant. Clearly, the major variables across the analyses were target's sex and vertical ratio, and a numerical comparison between the $\beta$ s suggested that target's sex was more effective than vertical ratio.

Table 8 shows the results of the clothes condition. For the analysis using the five variables, the obtained $R^{2}$ was .811 , and the $\beta$ s for sex $[-.521, t(44)=5.68, p<.001]$ and vertical ratio $[-.304, t(44)=4.11, p<.001]$ were significant. For the analyses using four variables, the highest $R^{2}$ we obtained from the four possible models was .806 , and the $\beta$ s for sex $[-.549, t(45)=6.20, p<.001]$, area ratio $[-.213, t(45)=2.28, p<.05]$, and vertical ratio $[-.303, t(45)=4.09, p<.001]$ were significant. For the analyses using three variables, the highest $R^{2}$.we obtained from the six possible models was .797 , and the $\beta$ s for $\operatorname{sex}[-.553, t(46)=6.17, p<.001]$, area ratio $[-.222$, $t(46)=2.36, p<.05]$, and vertical ratio $[-.310, t(46)=$ $4.14, p<.001]$ were significant. These results indicated that target's sex and vertical ratio were constantly effective and area ratio was partially effective.

Overall, the $R^{2}$ obtained by the regression analyses decreased as the target pictures were degraded. For example, the $R^{2} \mathrm{~s}$ for the five predictors were $.811, .786$, and .701 for the clothes, face, and silhouette conditions, respectively. This implies that, for greatly degraded drawings, the variables examined in this study might not be sufficient to be good predictors.

The effects of target's sex were strong through all degradations: In any degradation, the $\beta$ s for target's sex were larger than -.50 . These results agreed with those of the photographed persons.

The effects of body proportions depended on degradation: In the face and clothes conditions, vertical ratio was mildly effective, whereas in the silhouette condition, vertical ratio was not effective and was supplanted by area ratio and fit. Since the contours of the faces in the sil- houette condition were erased partially, as shown in Figure 5 , it may be difficult for the subjects to perceive the face heights accurately. Figure 7 shows the relations between mean estimates and vertical ratio for each degradation. Clearly, the simple correlation between them decreased as the pictures were degraded.

One may be surprised at the accurate estimation for the degraded drawings and may have a question as to how the accurate estimates were obtained when many visual features of target's persons were removed. One source is the recognition of the target's sex: The rate of correct sex recognition was more than $91 \%$, even for the silhouette drawings. The contours of hair and clothes probably supply the subjects with information about the target's sex. For example, a silhouette of long hair gives a hint that it may be a woman, whereas a silhouette of pants is an allusion that it may be a man.

However, it is not sufficient to account for the accurate estimation exclusively by recognition of target's sex. What were other sources in the silhouette condition, in which any ratio of head to trunk was not found to be effective? One possible source, which was not examined in this study but may contribute to height estimates, is the stubbiness of trunk (i.e., the ratio of shoulder width to shoulder height): A stubby person may be short of stature. It may also be assumed that the center of gravity has a correlate with real full height. However, we do not know how these variables are really effective on height estimates. It is thus worthwhile to assess the effects of these variables in future studies.

\section{GENERAL DISCUSSION}

A main finding of this study is the high correlation between mean estimated height and real height. It was shown that the correlation was .92 for the upright photographs and was .80 even for the contour drawings that were greatly reduced from the realistic person pictures. Accordingly, we may correctly estimate the height of persons at far dis-

\section{Table 8}

Squared Multiple Regression Coefficient $\left(R^{2}\right)$ and $\beta$ Weights Obtained for the Height Estimates of the Clothes Drawings

\begin{tabular}{lcccccc}
\hline & & \multicolumn{5}{c}{ Predictors } \\
\cline { 3 - 7 } $\mathrm{NP}$ & $R^{2}$ & Sex & Area & Hor. & Ver. & Fit \\
\hline 5 & .811 & $-.521 \ddagger$ & -.190 & -.089 & $-.304 \ddagger$ & -.085 \\
4 & .794 & $-.614 \ddagger$ & & -.123 & $-.350 \ddagger$ & -.089 \\
4 & .806 & $-.549 \ddagger$ & $-.213^{*}$ & & $-.303 \ddagger$ & -.096 \\
4 & .738 & $-.544 \ddagger$ & $-.312 \dagger$ & -.085 & & -.103 \\
4 & .804 & $-.519 \ddagger$ & $-.194^{*}$ & -.104 & $-.310 \ddagger$ & \\
3 & .733 & $-.571 \ddagger$ & $-.334 \dagger$ & & & -.114 \\
3 & .787 & $-.615 \ddagger$ & & -.139 & $-.357 \ddagger$ & \\
3 & .728 & $-.543 \ddagger$ & $-.319 \dagger$ & -.103 & & \\
3 & .797 & $-.553 \ddagger$ & $-.222^{*}$ & & $-.310 \ddagger$ & \\
3 & .688 & $-.720 \ddagger$ & & -.146 & & -.115 \\
3 & .783 & $-.671 \ddagger$ & & & $-.356 \ddagger$ & -.106 \\
\hline
\end{tabular}

Note-NP, number of predictors; Hor., horizontal ratio; Ver., vertical ratio. Significance of each predictor was evaluated with $t$ tests $(d f=50-$ $\mathrm{NP}-1) . \quad{ }^{*} p<.05 . \quad{ }^{\dagger} p<.01 . \quad{ }^{\ddagger} p<.001$. 

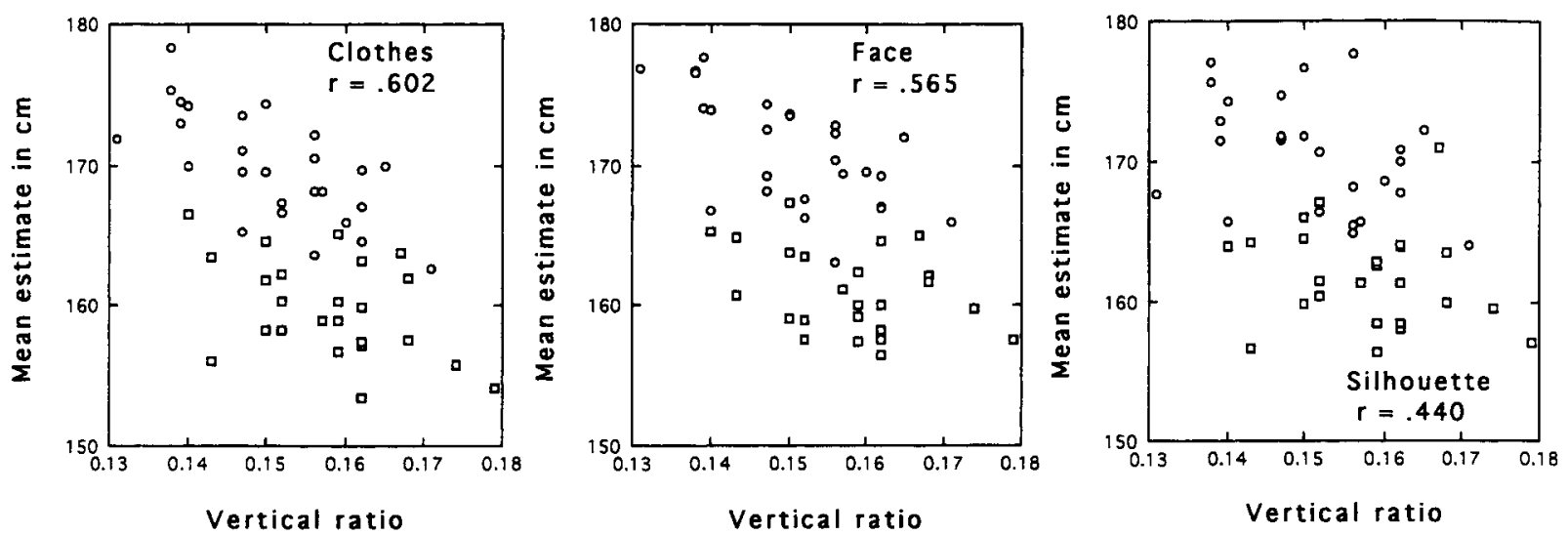

Figure 7. Mean height judgments for the depicted persons (i.e., clothes, face, and silhouette) as a function of vertical ratio (i.e., ratio of head height to full body height). Circles represent men, and squares represent women.

tances at which the conventional cues to distance are eliminated and the visual detail of target persons is obscured.

The correlations between mean estimated height and real height were clearly lower for the inverted and degraded pictures than for the upright photographs. This decrease in correlation was prominent for female targets. Table 9 summarizes the correlations between mean estimated height and real height separately for male, female, and overall targets. Interestingly, the correlation for female targets was lower than that for male targets in any picture condition, and this tendency was remarkable for the degraded drawings. This implies that, if persons are recognized to be women and if their detail is not perceived sufficiently, their heights cannot be discriminated, whereas, if the persons are recognized to be men, their heights are likely to be discriminated, even in poor visual conditions. In short, male targets confer a stronger metric of visual space than do female targets.

Why did we obtain the low correlations for the degraded female drawings? One possible explanation is that the degraded female targets were likely to be mistaken for males, as is indicated by the results of Experiment 3 . In particular, errors frequently occurred for the female targets who put on pants and had short hair, because there seems to be another belief that men more often put on pants and have shorter hair than do women. On the other hand, most male targets were identified correctly, probably because no male targets put on skirts or had long hair.

The results of the regression analyses suggested that the height estimates were mainly determined by target's sex and vertical ratio. In particular, the effects of target's sex were strong and consistent in all conditions in this study. The mean $\beta$ s taken across the 11 models for the upright photographs, inverted photographs, contour drawings, face drawings, and clothes drawings were -.630 , $-.855,-.571,-.658$, and -.584 , respectively.

The effects of vertical ratio interacted with degradation of target pictures: When detail of target persons was available, vertical ratio was effective on height estimates, but when their detail was largely lost (i.e., the silhouette condition), the effects of vertical ratio were not found and were replaced by the effects of fit. Probably because of the scant information that was delivered from the silhouette drawings, the subjects had to rely on the belief that tight-fitting clothes make the body appear taller than do loose-fitting clothes. However, it seems that this belief was not as strong as the belief that men are taller than women, because (1) the effects of fit were found only for the silhouette drawings and (2) the mean $\beta$ s for fit were not as large as the $\beta$ s for sex and vertical ratio.

The effects of area ratio and horizontal ratio were also found in several conditions: The effects of area ratio were found in the silhouette and clothes conditions, and the effects of horizontal ratio were found only in the upright photograph condition. Because these effects were found sporadically, it may safely be concluded that the effects of area ratio and horizontal ratio were not stable.

It seems that vertical ratio, rather than area ratio and horizontal ratio, is indeed an ecologically valid cue. If area ratio and horizontal ratio were effective on height estimates, our height estimates for a person would have to vary with the clothes the person wears. For example, if the person wears loose-fitting clothes, such as an overcoat, the area ratio and horizontal ratio decrease, because the area of trunk seemingly swells. Similarly, if the person wears tight-fitting clothes, such as a swimming suit, these ratios increase, as compared with those in everyday wear. However, vertical ratio is invariant, regardless of the

Table 9

Summary of Simple Correlations Between Mean Height Judgment and Real Height

\begin{tabular}{lccc}
\hline & \multicolumn{3}{c}{ Target's Sex } \\
\cline { 2 - 4 } \multicolumn{1}{c}{ Condition } & Men & Women & Overall \\
\hline Upright photographs & $.82 \dagger$ & $.80 \dagger$ & $.92 \dagger$ \\
Inverted photographs & $.79 \dagger$ & $.64 \dagger$ & $.88 \dagger$ \\
Clothes drawings & $.67 \dagger$ & .39 & $.82 \dagger$ \\
Face drawings & $.40^{*}$ & .35 & $.76 \dagger$ \\
Silhouette drawings & $.69 \dagger$ & .34 & $.80^{\dagger}$ \\
\hline$* p<05 .+$ & & &
\end{tabular}


clothes the person wears. Thus, the use of vertical ratio would have the merit of estimating person height correctly.

Our results may be comparable with the work of Bingham (1993a, 1993b), who studied how trees provide information about the scale of visual space. The correlation between estimated height and actual height was found to be about .77 for photographed trees. This value was larger than zero but was smaller than the correlation for the photographed upright persons. Thus, although it is clear that both persons and trees are available as metric sticks of visual space, persons may provide more veridical information than do trees.

Another advantage of using persons as a metric stick is that several artificial objects also provide us with information of the absolute metric of visual space. The everyday artificial objects around us are made so as to fit to our whole bodies or various body parts. For example, houses, cars, and beds are made relative to the full size of persons, and books, keyboards, and pens are made relative to the size of human hands and fingers. Therefore, if, as is shown in this study, we estimate the full body height of persons correctly, we are also successful in estimating the sizes of these common products.

There are several limitations to using persons as a metric stick. First, when we face the scenes in which there are no persons or no artificial objects, we could not use the person height as a metric stick. In these situations, we may rely on natural objects, such as trees, to obtain information of absolute metric. Second, for relatively large objects, such as towers, valleys, and mountains, the person height may not be useful, because it is very small for a tool of measurement. We do not know what provides the absolute scale for the huge objects.

\section{REFERENCES}

Alley, T. R. (1983). Age-related changes in body proportions, body size, and perceived cuteness. Perceptual \& Motor Skills, 56, 615-622.

Bingham, G. P. (1993a). Perceiving the size of trees: Biological form and the horizon ratio. Perception \& Psychophysics, 54, 485-495.

Bingham, G. P. (1993b). Perceiving the size of trees: Forms as information about scale. Journal of Experimental Psychology: Human Perception \& Performance, 19, 1139-1161.

CARLson, V. R., \& TAssone, E. P. (1971). Familiar versus unfamiliar size: A theoretical derivation and test. Journal of Experimental Psychology, 87, 109-115.

Fillembaum, S., Schiffman, H. R., \& Butcher, J. (1965). Perception of off-size versions of a familiar object under conditions of rich information. Journal of Experimental Psychology, 69, 298-303.

Fitzpatrick, V., Pasnak, R., \& Tyer, Z. E. (1982). The effect of familiar size at familiar distances. Perception, 11, 85-91.
GogeL, W. C. (1969). The effect of object familiarity on the perception of size and distance. Quarterly Journal of Experimental Psychology, 21, 239-247.

GogEL, W. C. (1981). The role of suggested size in distance responses. Perception \& Psychophysics, 30, 149-155.

GogeL, W. C., \& DA Silva, J. A. (1987a). Familiar size and the theory of off-sized perceptions. Perception \& Psychophysics, 41, 318-328.

GoGel, W. C., \& DA Silva, J. A. (1987b). A two-process theory of the response to size and distance. Perception \& Psychophysics, 41, 220-238.

Higashiyama, A. (1982). The effects of familiar-size cue on children's judgments of size and distance. Japanese Journal of Psychology, 53, 259-265.

Higashiyama, A. (1984). The effects of familiar size on judgments of size and distance: An interaction of viewing attitude with spatial cues. Perception \& Psychophysics, 35, 305-312.

HigashiYama, A. (1996). Horizontal and vertical distance perception: The discorded-orientation theory. Perception \& Psychophysics, 58, 259-270.

Higashiyama, A., \& Kitano, S. (1991). Perceived size and distance of persons in natural outdoor settings: The effects of familiar size. $P s y$ chologia, 34, 188-199.

Howard, I. P. (1982). Human visual orientation. Chichester, U.K.: Wiley.

KöHLER, W. (1940). Dynamics in psychology. New York: Liveright. LEIBowITZ, H. W., \& HARVEY, H. O., JR. (1967). Size matching as a function of instructions in a naturalistic environment. Journal of Experimental Psychology, 74, 378-382.

LeibowitZ, H. W., \& HARVEY, H. O., JR. (1969). Effect of instructions, environment, and type of test object on matched size. Journal of Experimental Psychology, 81, 36-43.

MEDAWAR, P. B. (1944). The shape of the human being as a function of time. Proceedings of the Royal Society of London: Series B, 132, 133 141.

Nelson, T. E., Biernat, M. R., \& Manis, M. (1990). Everyday base rates (sex stereotypes): Potent and resilient. Journal of Personality \& Social Psychology, 59, 664-675.

PitTenger, J. B. (1990). Body proportions as information for age and cuteness: Animals in illustrated children's books. Perception \& Psychophysics, 48, 124-130.

Pittenger, J. B., \& Todd, J. T. (1983). Perception of growth from changes in body proportions. Journal of Experimental Psychology: Human Perception \& Performance, 9, 945-954.

Predebon, J. (1992). The role of instructions and familiar size in absolute judgments of size and distance. Perception \& Psychophysics, 51, 344-354.

Predebon, J. (1994). Perceived size of familiar objects and the theory of off-sized perceptions. Perception \& Psychophysics, 56, 238-247.

Rock, I. (1973). Orientation and form. New York: Academic Press.

Rock, I. (1988). On Thompson's inverted-face phenomenon. Perception, 17, 815-817.

Rock, I., \& Kaufman, L. (1962). The moon illusion, II. Science, 136, 1023-1031.

Todd, J. T., Mark, L. S., Shaw, R. E., \& PitTenger, J. B. (1980, February). The perception of human growth. Scientific American, 242, 106-114.

(Manuscript received May 16, 1997; revision accepted for publication November $16,1997$. 